\title{
Intervenção ortocirúrgica em paciente adolescente com acentuada displasia esquelética de Classe III
}

\author{
Andressa Otranto de Britto Teixeira*, Paulo José Medeiros**, Jonas Capelli Junior***
}

\begin{abstract}
Resumo
Introdução: a má oclusão de Classe III é caracterizada por uma discrepância esquelética ântero-posterior, podendo ou não estar acompanhada de alterações verticais, mas comumente apresenta alterações transversais associadas. $\mathrm{O}$ aspecto facial fica comprometido nesses pacientes, sendo esse um dos fatores que os motivam a procurar o tratamento ortodôntico. Quando o paciente encontra-se na faixa de 8 a 10 anos de idade, uma abordagem precoce é indicada. Quando o paciente é adulto e o crescimento já cessou, o tratamento vai ser decidido entre a camuflagem ortodôntica e os procedimentos ortocirúrgicos clássicos. O problema quanto à decisão terapêutica está no paciente adolescente, com acentuadas Classes III esqueléticas, quando os procedimentos interceptativos não mais surtirão o efeito desejado e as alterações faciais são muito significativas. Ainda haverá crescimento e muitas vezes há comprometimento psicossocial ou funcional. Objetivo: propor uma abordagem cirúrgica precoce como alternativa de tratamento para esses pacientes, mesmo que uma segunda cirurgia seja necessária após o término do crescimento. Discussão: para se propor essa terapia, vários critérios devem ser observados para que realmente o paciente tenha benefícios com esta intervenção precoce, como pouca discrepância intra-arco e possibilidade de preparo ortodôntico pré-cirúrgico rápido. Conclusão: deve-se ter ciência que um segundo tratamento ortocirúrgico provavelmente se fará necessário após o término do crescimento e que esse tipo de tratamento não deve ser empregado como rotina.
\end{abstract}

Palavras-chave: Má oclusão de Classe III. Ortodontia. Cirurgia ortognática precoce.

\footnotetext{
* Especialista em Ortodontia ABO/JF. Mestranda em Ortodontia UERJ.
** Doutor em Cirurgia buco-maxilo-facial pela UFRJ. Pós-graduado em cirurgia Buco-maxilo-facial University of Texas, Dallas, EUA. Professor Titular Cirurgia Bucal FO-UFRJ. Chefe do Serviço de Buco-maxilo-facial HU-UERJ.

*** Livre Docente em Ortodontia pela UERJ. Professor Adjunto de Ortodontia da FO-UERJ. Diplomado pelo Board Brasileiro de Ortodontia.
} 


\section{INTRODUÇÃO}

A má oclusão de Classe III de origem esquelética é caracterizada por discrepância ântero-posterior entre as bases ósseas, que pode ser devida à deficiência da maxila, ao excesso mandibular ou à combinação de ambos. Estas anomalias levam a alterações expressivas do perfil facial do paciente, muitas vezes com conseqüências psicossociais

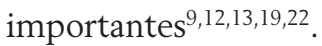

As terapias para tratamento da displasia estão relacionadas com o potencial do crescimento do indivíduo. Quando o paciente encontra-se na fase de 8 a 10 anos, ou seja, na fase pré-surto de crescimento puberal, uma abordagem precoce é indicada com uso de tração reversa da maxila, normalmente acompanhada de disjunção palatina. Com esta abordagem procura-se obter um incremento do tamanho da maxila, junto a uma rotação horária da mandíbula, conseguindo-se uma melhor relação entre esses ossos e uma oclusão satisfatória ${ }^{1,3,4,10,17,18}$. Quando o paciente é adulto e o crescimento já cessou, o tratamento vai ser decidido entre os procedimentos de camuflagem, caso a displasia não seja muito significativa, e os procedimentos ortocirúrgicos clássicos, podendo envolver avanço de maxila, recuo da mandíbula ou procedimentos combinados ${ }^{4}$. O problema quanto à decisão terapêutica está na abordagem das Classes III com envolvimento esquelético significativo na fase onde certamente a abordagem preventiva não mais surtirá efeito, mas ainda há crescimento.

\section{DIAGNÓSTICO E ETIOLOGIA}

Paciente R. P. D., gênero masculino, leucoderma, 14 anos e 9 meses, procurou tratamento na clínica de especialização em Ortodontia da Faculdade de Odontologia da UERJ, tendo como queixa principal o tamanho do queixo (Fig. 1, 2, 3), que julgava muito grande. Foi realizado exame clínico e solicitada documentação ortodôntica.

No exame clínico foi constatada relação de Classe III bilateral (Fig. 4, 5, 6), com overjet de $-3 \mathrm{~mm}$, overbite nulo e ausência de problemas intra-arcos significativos. $\mathrm{O}$ exame facial revelou um perfil característico de Classe III com protrusão mandibular.

Os exames radiográficos revelaram um padrão de Classe III com $\mathrm{ANB}=-4^{\circ}\left(\mathrm{SNA}=86^{\circ} / \mathrm{SNB}=90^{\circ}\right)$ e Wits de $-13 \mathrm{~mm}$ (Fig. 7).

\section{OBJETIVOS DE TRATAMENTO}

Oferecer ao paciente com deformidades faciais graves condições psicossociais de desenvolvimento durante a adolescência, melhorando funções como fonação e deglutição, além de promover grande melhora na sua estética facial. A abordagem cirúrgica precoce torna-se uma alternativa de tratamento para esse tipo de paciente, mesmo que uma segunda cirurgia se faça necessária após o término do crescimento ${ }^{14,15,20,21}$. Para se propor essa terapia vários critérios foram observados, para que realmente o paciente tivesse benefícios. Este, certamente, não é um modelo de tratamento que pode ser proposto para qualquer caso.

\section{ALTERNATIVAS DE TRATAMENTO}

A indicação formal de tratamento seria aguardar o fim do crescimento para poder se estabelecer um tratamento ortocirúrgico convencional ${ }^{5,6,7,8}$. Para tanto, é necessário que o paciente atravesse toda a fase da adolescência com uma deformidade facial que pode gerar uma condição estética seriamente comprometida, acarretando uma baixa aceitação social perante o seu meio.

\section{PROGRESSO DO TRATAMENTO}

Foi montado aparelho fixo do tipo Edgewise, com bandagem dos primeiros molares e colagem de braquetes em incisivos, caninos e pré-molares. Inicialmente foram colocados arcos de aço 0,016" e logo em seguida arcos de aço retangulares 0,019"x 0,025" passivos, com spürs para fixação no momento da cirurgia (Fig. 8, 9, 10). O período total de preparo pré-cirúrgico foi de 5 meses e o paciente foi imediatamente encaminhado ao Serviço de Cirurgia Bucomaxilofacial do Hospi- 


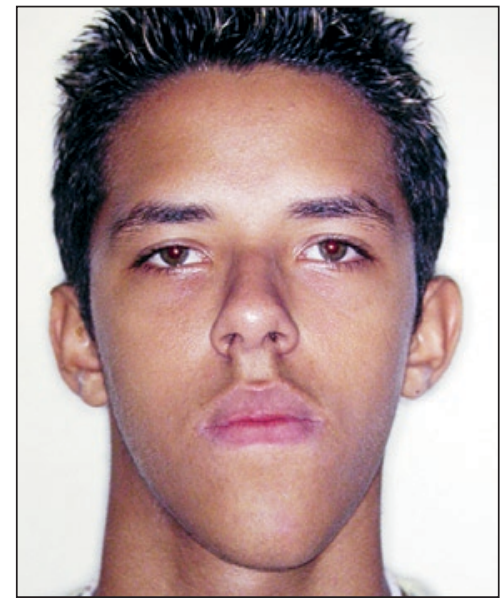

FIGURA 1 - Fotografia extrabucal frontal inicial.

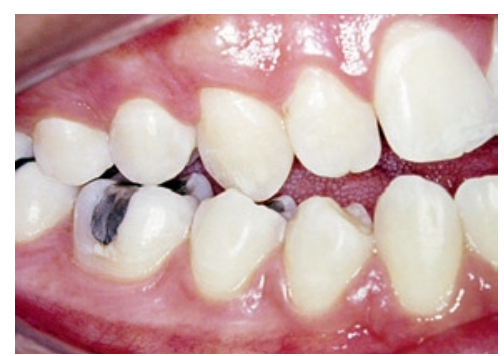

FIGURA 4 - Fotografia intrabucal lareral direita inicial.

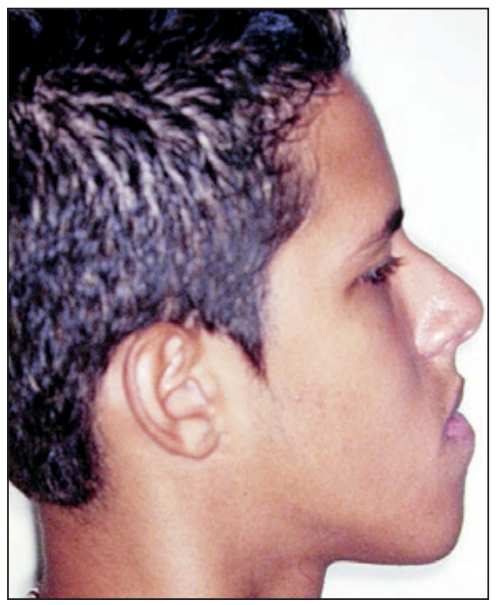

FIGURA 2 - Fotografia extrabucal perfil inicial.

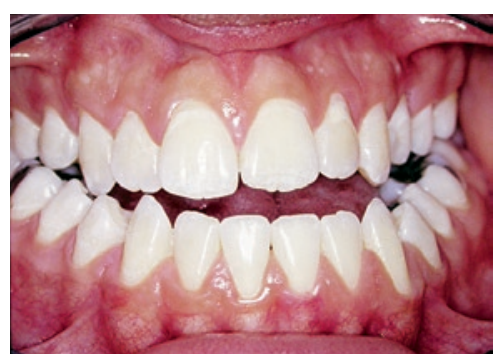

FIGURA 5 - Fotografia intrabucal frontal inicial.

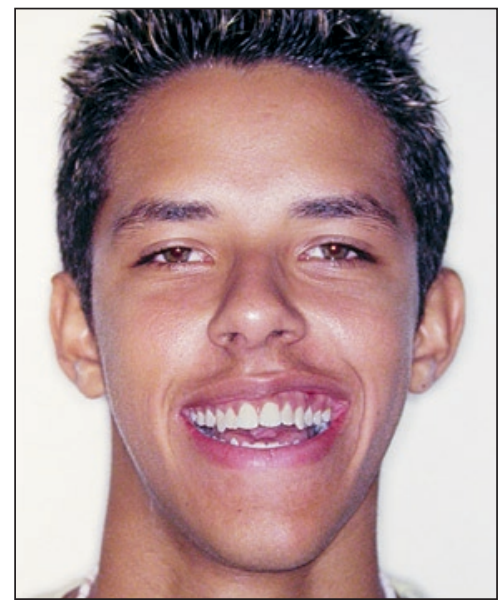

FIGURA 3 - Fotografia extrabucal sorrindo inicial.

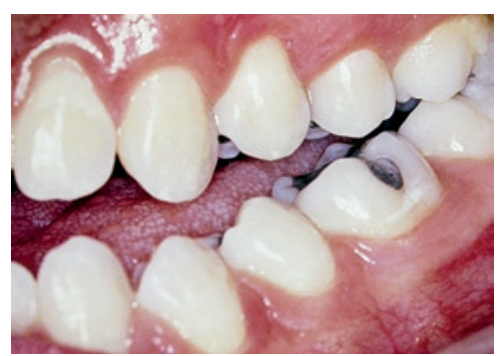

FIGURA 6 - Fotografia intrabucal lateral esquerda inicial. tal Universitário Pedro Ernesto (HUPE-UERJ) para procedimento cirúrgico. Após a avaliação da condição clínica do paciente, o mesmo foi submetido à cirurgia ortognática para redução mandibular, por meio de procedimento cirúrgico de osteotomia vertical do ramo mandibular ${ }^{11,13,16}$. Os procedimentos de finalização duraram 4 meses, contando com o período de fixação pós-cirúrgica. Após esse período, foi realizada remoção da aparelhagem fixa e colocação de contenção superior removivel do tipo wraparound ${ }^{8}$.

\section{RESULTADOS DO TRATAMENTO}

O paciente obteve um resultado pós-cirúrgico satisfatório, com obtenção de relação de Classe I de Angle, corretos overjet e overbite (Fig. 11 - 16).

No acompanhamento pós-cirúrgico, 12 meses

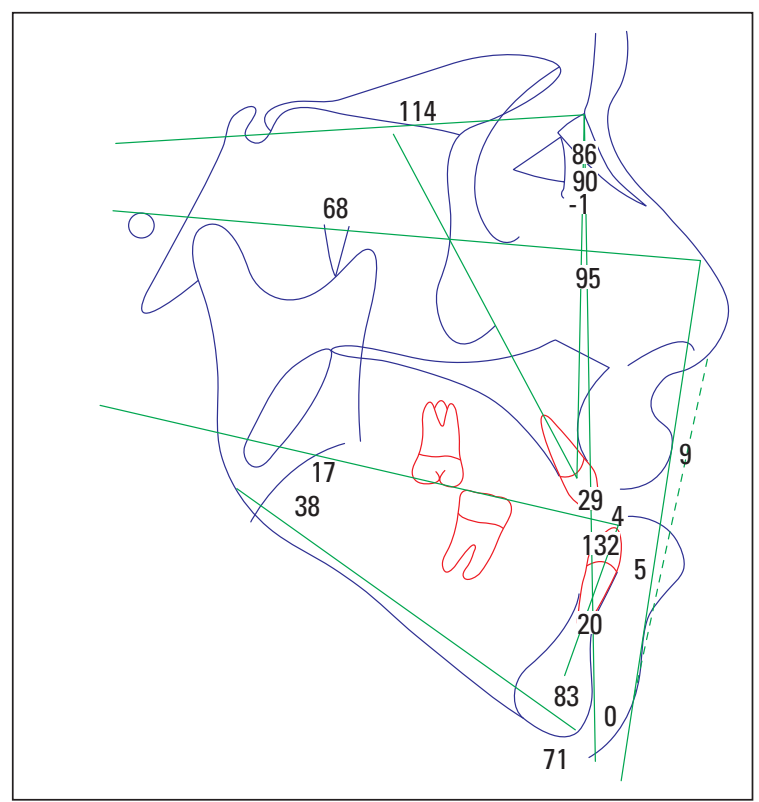

FIGURA 7 - Traçado cefalométrico inicial. 


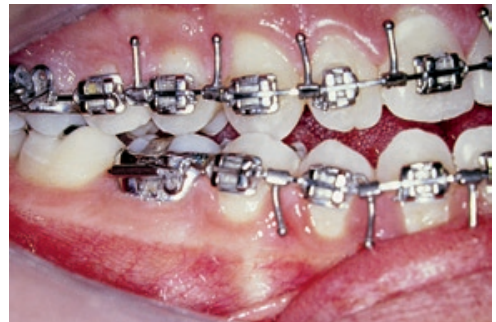

FIGURA 8 - Fotografia intrabucal lateral direita pré-cirúrgica.

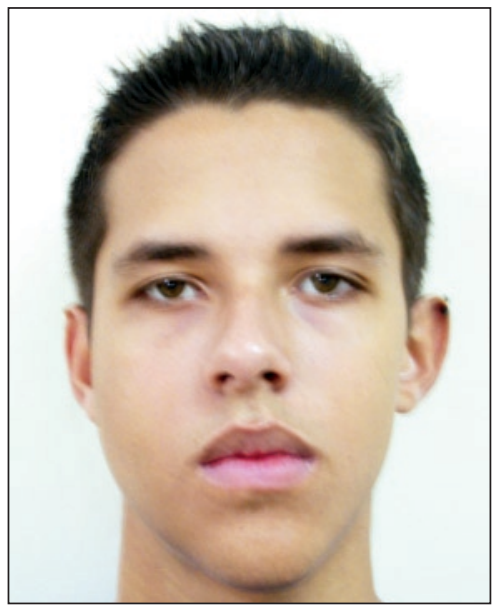

FIGURA 11 - Fotografia extrabucal frontal póscirúrgica.

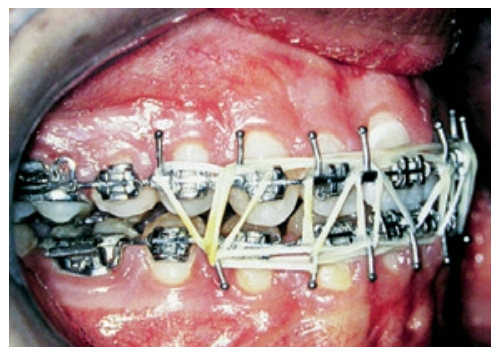

FIGURA 14 - Fotografia intrabucal lateral direita pós-cirúrgica.

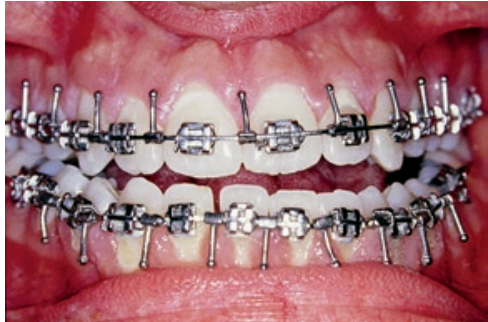

FIGURA 9 - Fotografia intrabucal frontal précirúrgica.

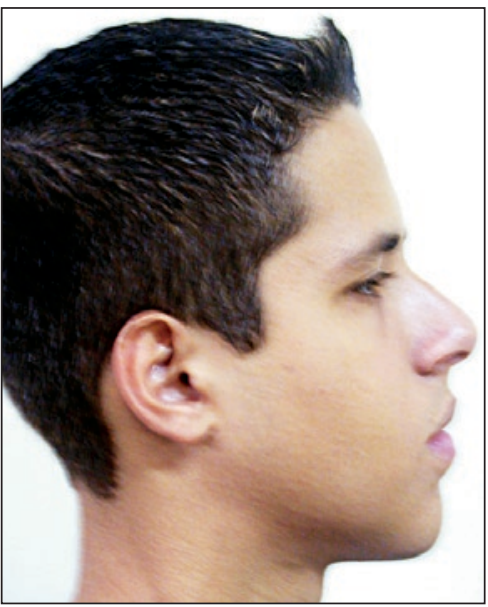

FIGURA 12 - Fotografia extrabucal perfil póscirúrgica.

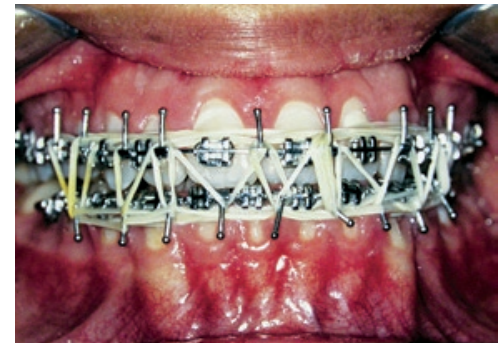

FIGURA 15 - Fotografia intrabucal frontal póscirúrgica.

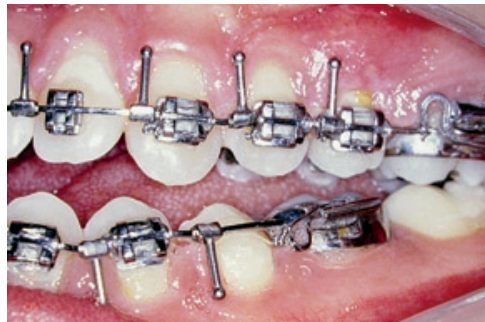

FIGURA 10 - Fotografia intrabucal lateral esquerda pré-cirúrgica.

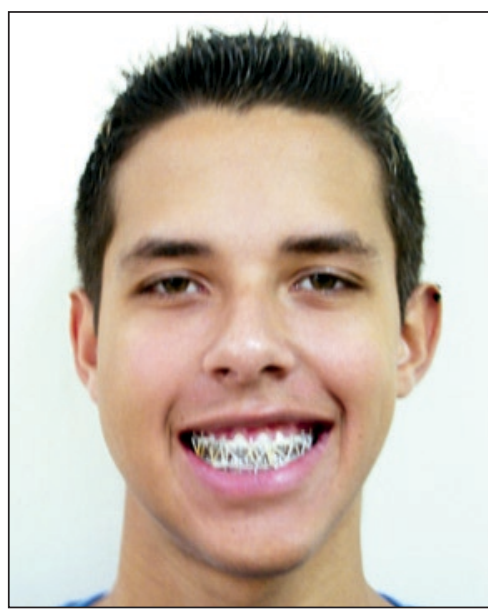

FIGURA 13 - Fotografia extrabucal frontal sorrindo pós-cirúrgica.

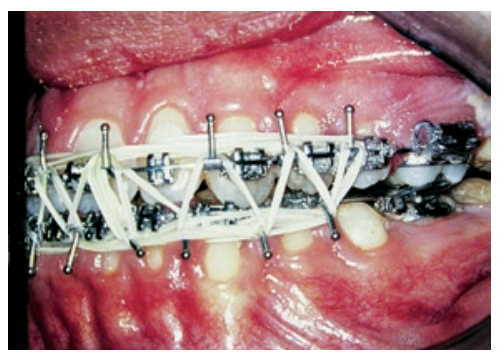

FIGURA 16 - Fotografia intrabucal lateral esquerda pós-cirúrgica. após a intervenção, verificou-se alterações oclusais, com diminuição do overjet e do overbite que apresentavam-se nulos, o que havia sido percebido pelo paciente. Aos 24 meses, o overjet havia se tornado negativo e após 3 anos da cirurgia, quando o paciente já encontrava-se com 18 anos de idade, o overjet apresentava-se ainda mais negativo, observando-se a necessidade de um segundo tratamento ortocirúrgico (Fig. 17 - 22).

$\mathrm{O}$ relato do paciente, sobre o tratamento ao qual foi submetido, foi de ter se sentido plenamente satisfeito com os resultados obtidos pela cirurgia precoce, mesmo sabendo da necessidade de uma nova intervenção, posteriormente, e dos incômodos pós-operatórios. Segundo ele, essa cirurgia mudou completamente a sua adolescência, pois ele se achava feio, tinha apelidos relacionados à sua deformidade facial, estava com a auto-estima abalada e não conseguia se relacionar com as pessoas. Após a cirurgia, diz que passou a se achar 


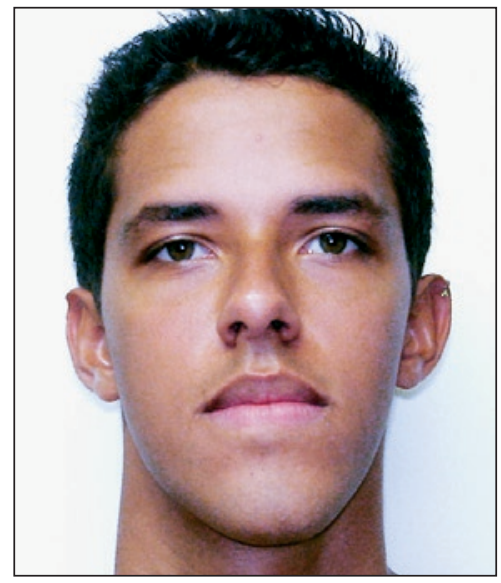

FIGURA 17 - Fotografia extrabucal frontal 3 anos após cirurgia (aos 18 anos).

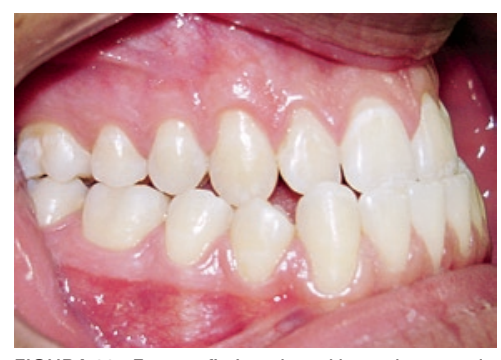

FIGURA 20 - Fotografia intrabucal lateral esquerda 3 anos após a cirurgia.

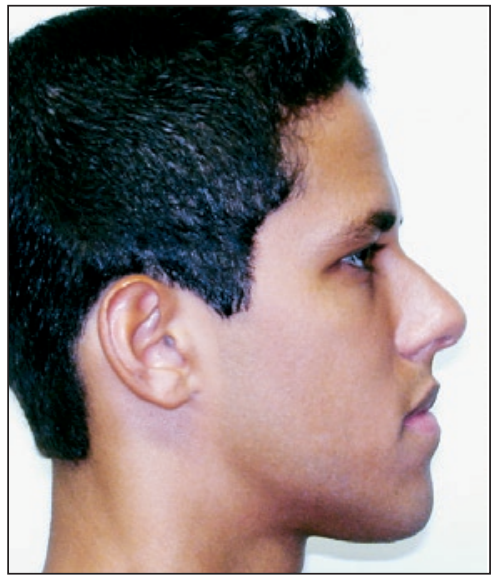

FIGURA 18 - Fotografia extrabucal perfil 3 anos após a cirurgia (aos 18 anos).

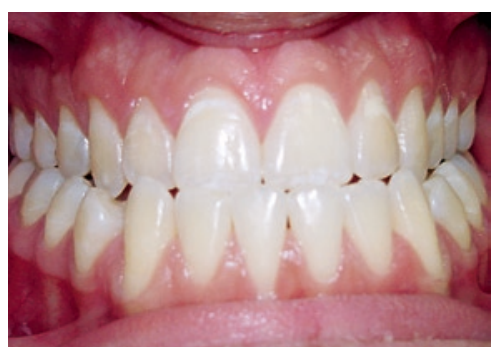

FIGURA 21 - Fotografia intrabucal frontal 3 anos após a cirurgia.

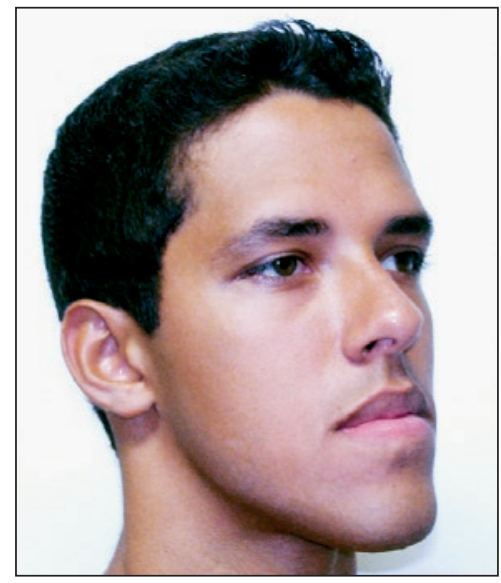

FIGURA 19 - Fotografia extrabucal $45^{\circ} 3$ anos após a cirurgia (aos 18 anos).

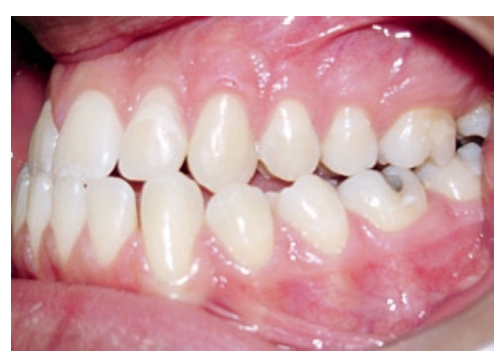

FIGURA 22 - Fotografia intrabucal lareral direita 3 anos após a cirurgia. bonito e a conseguir se relacionar bem com as pessoas. Afirma que para ele o procedimento foi válido e que voltaria a optar, novamente, por esse tratamento.

\section{DISCUSSÃO}

A má oclusão de Classe III tem recebido especial atenção dos ortodontistas, desde as primeiras publicações que relataram este desvio da normalidade. A má oclusão de Classe III é caracterizada por uma discrepância esquelética ântero-posterior, podendo ou não estar acompanhada por alterações verticais, mas comumente com alterações transversais associadas. Estas discrepâncias esqueléticas provocam alterações no posicionamento dos dentes inter e intra-arcos. $\mathrm{O}$ aspecto facial fica comprometido nesses pacientes, sendo justamente esse fator que os motiva a procurar tratamento para esse tipo de má oclusão ${ }^{12,13}$.
A grande variação de expressão desta má oclusão e sua relação com alterações no crescimento e desenvolvimento craniofaciais sempre levantaram muitas dúvidas quanto à época correta de se intervir e a correta mecânica a ser empregada. Dentre as diversas modalidades terapêuticas sugeridas na literatura encontramos o uso de elásticos intermaxilares, as extrações dentárias, aparelhos ortopédicos intra e extrabucais e tratamentos ortocirúrgicos ${ }^{4}$, normalmente com indicações específicas para a fase de desenvolvimento em que se encontra o paciente.

Durante muito tempo acreditou-se que o potencial de crescimento inerente a cada indivíduo seria um fator etiológico tão intenso nos casos de Classe III que qualquer tentativa de modificação do padrão de crescimento estaria fadada ao insucesso. Nas últimas décadas, com o sucesso de terapias como o uso de mentoneiras e, principalmente, 
o uso da tração reversa para protração maxilar, esses dogmas em relação à possibilidade de intervenções ortopédicas têm sido derrubados ${ }^{17}$. Hoje, a terapia de tração da maxila é um procedimento extremamente divulgado e aceito para a fase de pré-surto de crescimento puberal, quando o paciente encontra-se em torno dos 8 a 10 anos e apresenta uma displasia esquelética leve ou moderada ${ }^{3,10,17,18}$. Porém, a passagem por essa fase sem tratamento e uma tendência de crescimento desfavorável podem levar os pacientes a uma indicação de tratamento com terapias ortocirúrgicas associadas, para a obtenção de resultados satisfatórios ${ }^{4,7}$. Para a correta seleção e indicação dos casos a serem tratados por essa modalidade terapêutica é importante realizar-se análises faciais, cefalométricas, oclusais e funcionais. Outros fatores, como expectativa do paciente, sua motivação e seu perfil psicológico, complementam as informações para a correta indicação do caso para a cirurgia ${ }^{8,19}$. A terapia ortocirúrgica é indicada para uma fase mais tardia, normalmente cerca de 1 ano e meio após o término do surto de crescimento, devido à tendência de crescimento tardio da mandíbula nesses pacientes ${ }^{1,5}$. Nesse tipo de tratamento, os objetivos ortodônticos são as descompensações dentárias, o correto posicionamento dos dentes intra-arcos e o estabelecimento de condições interarcos que possibilitem o estabelecimento de uma correta oclusão e a fixação dos dentes e ossos durante o ato cirúrgico, e que permaneçam estáveis na fase pós-cirúrgica ${ }^{8,12,13}$. O que fazer, então, quando o paciente já passou pela fase em que a terapia ortopédica seria indicada, mas ainda encontra-se com potencial de crescimento ativo, e uma grande deformidade facial está presente, trazendo comprometimentos psicológicos para ele.

A terapia mais aceita na atualidade é aguardar o crescimento cessar e proceder com um tratamento ortocirúrgico convencional ${ }^{2}$. Entretanto, a tentativa de se realizar uma intervenção precoce pode ser considerada em casos onde a deformidade é acentuada e o comprometimento psicossocial significativo.
Em um estudo, a osteotomia do tipo Le Fort I, em indivíduos com excesso vertical, foi realizada em 48 pacientes, para impacção de maxila. Foi avaliada a fixação rígida em 23 pacientes e fixação esquelética com fios em 25 pacientes. Os autores ${ }^{14}$ verificaram que este tipo de cirurgia teve pouco ou nenhum efeito sobre o crescimento vertical da maxila, posteriormente à cirurgia, ou seja, a cirurgia não paralisou o crescimento, nem mesmo mudou o padrão de crescimento dos pacientes. Por isso afirmaram ser muito importante avisar ao paciente e familiares que uma nova cirurgia poderá se fazer necessária para corrigir novamente o problema.

Em relação às cirurgias precoces de maxila, a avaliação da impacção cirúrgica realizada em pacientes em crescimento não demonstrou efeito prejudicial no crescimento mandibular ou na altura facial. A impacção precoce da maxila não normalizou ou inibiu o padrão de crescimento vertical e o crescimento vertical pós-cirúrgico foi similar aos indivíduos com padrão vertical não tratados, mas foi diferente dos não tratados e com crescimento normal ${ }^{15}$.

Quanto às cirurgias para correção das deformidades mandibulares existem três opções, em relação à época do tratamento cirúrgico do paciente em crescimento com hiperplasia mandibular: adiar a cirurgia até o crescimento estar completado, mesmo considerando que possa haver problemas funcionais, com prejuízos na mastigação e fonação, desfiguramento estético, dor e estigmas psicossociais associados à deformidade facial severa; executar a cirurgia de recuo mandibular, com sobrecorreção, durante a fase de crescimento, pois o crescimento acelerado é esperado após a cirurgia e novo procedimento será necessário se a sobrecorreção for insuficiente ou excessiva; ou eliminar cirurgicamente o crescimento mandibular subseqüente com a condilectomia alta e, simultaneamente, corrigir a deformidade mandibular, sendo esta a opção preferida por alguns autores ${ }^{20}$.

Pacientes adolescentes com deformidades 
dentofaciais podem receber tratamento cirúrgico durante a fase de crescimento ativo, devido a problemas funcionais, de estética e fatores psicossociais. O conhecimento do crescimento facial, das opções de tratamento e dos efeitos da cirurgia no padrão de crescimento pós-operatório irá ajudar o ortodontista a melhorar seus cuidados com estes pacientes ${ }^{15,20,21}$. Mesmo em casos onde questões como as sugeridas pelos autores estejam presentes, são necessárias ainda condições específicas de oclusão que permitam que os princípios relacionados a esse tipo de terapia sejam respeitados. Esses princípios dizem respeito a uma possibilidade de preparo pré-cirúrgico rápido, com movimentação dentária mínima, pois se a fase pré-cirúrgica for longa, provavelmente, vai se aproximar do final do surto de crescimento, o que não justificaria mais uma cirurgia precoce. Além disso, a grande movimentação dentária no preparo pré-cirúrgico também teria um alto custo biológico, para depois se instituir uma nova terapia, que freqüentemente se faz necessária.

O resultado cirúrgico deve objetivar o melhor resultado estético e funcional para a época do tratamento, não considerando a sobrecorreção, já que a estimativa do crescimento residual é incerta. $\mathrm{O}$ paciente e os responsáveis devem ser alertados sobre a necessidade, quase certa, de um tratamento ortocirúrgico convencional após o final do crescimento maxilomandibular.

\section{CONCLUSÕES}

A cirurgia precoce para tratamento da Classe III pode ser indicada quando o paciente estiver com grande comprometimento psicossocial ou funcional.

Para que essa terapia possa ser indicada critérios ortodônticos, como pouca discrepância intraarco e possibilidade de preparo pré-cirúrgico sem grandes recolocações dentárias, devem ser observados.

Um segundo tratamento ortocirúrgico provavelmente se fará necessário após o término do crescimento.

Esse tratamento não deve ser considerado como tratamento de rotina, mas sim como uma possibilidade terapêtutica para casos criteriosamente selecionados.

\section{COMENTÁRIO DO EDITOR}

A clássica abordagem terapêutica da Classe III com acentuada displasia esquelética por aumento mandibular é feita após o fim do crescimento. A realização do tratamento precoce, como apresentada pelos autores, somente deve ser considerada quando o impacto psicossocial ou funcional do problema é danoso a ponto de justificar duas cirurgias - uma precoce e outra ao final do crescimento. Os autores posicionam essa importante questão com muita clareza e salientam os critérios necessários para a realização desse tipo de tratamento. A forma pouco usual de tratamento e a excelente argumentação dos autores fazem esse relato de caso merecer uma leitura minuciosa. 


\title{
Orthodontic-surgical treatment in youthful patient with severe skeletal Class III malocclusion
}

\begin{abstract}
Introduction: Class III malocclusion is characterized by an anterior-posterior skeletal discrepancy that may or may not be combined with vertical alterations, but it commonly presents transverse disharmony associated. The facial aspect is affected in these patients, which is one of the factors that motivate them to look for orthodontic treatment. For young patients (8 to 10 years old), an earlier treatment is indicated. In adult patients, when growth has already finished, the options of treatment usually are orthodontic camouflage or ortho/surgery traditional treatment. The question is how to treat adolescents patients with severe skeletal Class III discrepancies, when an interceptative procedure has no indication and facial modifications are very significant. They are still growing and there is, in some cases, psicosocial and functional implications. Objective: To consider an early surgical approach as alternative treatment for these patients; even if a second surgery is needed when growth is completed. Discussion: To indicate this therapy, some factors should be observed: small dentoalveolar discrepancies and rapid pre-surgical preparation. Conclusion: The patient must be informed of the possibility of another surgery at the end of the growth. This strategy of treatment should not be used as routine.
\end{abstract}

Key words: Class III malocclusion. Orthodontic. Early orthognathic surgery.

\section{REFERÊNCIAS}

1. ATHANADIOU, E. A. Morphologic and functional implications of the surgical-orthodontic management of mandibular prognathism: a comprehensive review. Am. J. Orthod. Dentofacial Orthop., St. Louis, v. 103, no. 5, p. 439-447, May 1997.

2. CARDOSO, M. A.; CAPELLI JR., J.; MEDEIROS, P. J. Tratamento orto-cirúrgico de pacientes com acentuada displasia esquelética de Classe III. R. Dental Press Ortodon. Ortop. Facial, Maringá, v. 9, n. 1, p. 137-144, jan./fev. 2004.

3. CARLINI, M. G.; MIGUEL, J. A. M.; GOLDNER, M. T. A. Tratamento precoce da má oclusão Classe III de Angle com expansão rápida e uso de máscara facial: relato de um caso clínico. R. Dental Press Ortodon. Ortop. Facial, Maringá, v. 7 , n. 2, p. 71-75, mar./abr. 2002.

4. CREEKMORE, T. D. Class III treatment planning. J. Clin. Orthod., Boulder, v. 12, no. 9, p. 650-655, Sept. 1978.

5. FISH, L. C.; EPKER, B. N. Prevention of relapse in surgicalorthodontic treatment. Part 1: mandibular procedures. J. Clin. Orthod., Boulder, v. 20, no. 12, p. 826-841, Dec. 1986.

6. FISH, L. C.: EPKER, B. N. Prevention of relapse in surgical-orthodontic treatment. Part 2: maxillary advancement and inferior repositioning. J. Clin. Orthod., Boulder, v. 21, no. 2, p. 100-108, Feb. 1987.

7. GRABER, T. M. Ortodontia: princípios e técnicas atuais. 2. ed. Rio de Janeiro: Guanabara Koogan, 1996.

8. JACOBS, J. D.; SINCLAIR, P. M. Principles of orthodontic mechanics in orthognathic surgery cases. Am. J. Orthod. Dentofacial Orthop., St. Louis, v. 84, no. 5, p. 399-407, Nov 1983.

9. JACOBSON, A. et al. Mandibular prognathism. Am. J. Orthod. Dentofacial Orthop., St. Louis, v. 66, no. 2, p. 140-171, Aug. 1974.

10. KIM, J. H. et al. The effectiveness of protraction facemask therapy: a meta-analysis. Am. J. Orthod. Dentofacial Orthop., St. Louis, v. 115, no. 6, p. 675-685, June 1999

11. MEDEIROS, P. J. Avaliação funcional de pacientes submetidos a recuo mandibular através da osteotomia vertical do ramo. 1999. 63 f. Tese (Doutorado)-Universidade Federal do Rio de Janeiro, Rio de Janeiro, 1999.

12. MEDEIROS, P. J. Cirurgia ortognática. In: ZANINI, S. Cirurgia e traumatologia bucomaxilofacial. Rio de Janeiro: Revinter, 1990. p. 251-320.

13. MEDEIROS, P. J.; MEDEIROS, P. P. Cirurgia ortognática para o ortodontista. 2. ed. São Paulo: Ed. Santos, 2004.
14. MOGAVERO, F. J.; BUSCHANG, P. H.; WOLDORF, L. M. Orthognathic surgery effects on maxillary growth in patients with vertical maxillary excess. Am. J. Orthod. Dentofacial Orthop. St. Louis, v. 111, no. 3, p. 288-296, Mar. 1997.

15. MOJDEHI, M.; BUSCHANG, P. H.; ENGLISH, J. D.; WOLDORF L. M. Post surgical growth changes in the mandible of adolescents with vertical maxillary excess growth pattern. Am. J. Orthod. Dentofacial Orthop., St. Louis, v. 119, no. 2, p. 106116, Feb. 2001

16. PROFFIT, W. R. Ortodondia contemporânea. 2. ed. Rio de Janeiro: Guanabara Koogan, 1995.

17. SILVA FILHO, O. G.; MAGRO, A. C.; OZAWA, T. O. Má oclusão de Classe III: caracterização morfológica na infância: dentadura decídua e mista. Ortodontia, São Paulo, v. 30, n. 2, p. 7-20, maio/ago. 1997.

18. SILVA FILHO, O. G.: MAGRO, A. C.: CAPELOZZA FILHO, L. Early treatment of the Class III malocclusion with rapid maxillary expansion and maxillary protraction. Am. J. Orthod. Dentofacial Orthop., St. Louis, v. 113, no. 2, p. 196-203, Feb. 1998.

19. WARREN, D. W. Keys to treatment plans for Class III patients with skeletal discrepancies. J. Clin. Orthod., Boulder, v. 24 no. 6, p. 370-374, June 1990

20. WOLDORF, L. M.; KARRAS, S. C.; MEHRA, P. Considerations for orthognathic surgery during growth. Part 1: mandibular deformities. Am. J. Orthod. Dentofacial Orthop., St. Louis, v. 119, no. 2, p. 95-101, Feb. 2001

21. WOLDORF, L. M.; KARRAS, S. C.; MEHRA, P. Considerations for orthognathic surgery during growth. Part 2: maxillary deformities. Am. J. Orthod. Dentofacial Orthop., St. Louis, v. 119, no. 2, p. 102-105, Feb. 2001

22. WOODS, M. G.; SWIFT, J. Q.; MARKOWITS, N. R. Clinical implications of advances in orthognatic surgery. J. Clin. Orthod., Boulder, v. 23, no. 6, p. 420-429, June 1989.

\section{Endereço para correspondência}

Andressa Otranto de Britto Teixeira

Rua Avenida Assunção 624 - Centro

CEP: 28.906-200 - Cabo Frio / RJ

E-mail: andressaotranto@terra.com.br 